\title{
INTEGRATED VERTICAL PARALLEL PLATE CAPACITIVE HUMIDITY SENSOR
}

\author{
N. Lazarus* ${ }^{*}$ and G. K. Fedder
}

Department of Electrical and Computer Engineering and the Institute for Complex Engineered Systems

Carnegie Mellon University, Pittsburgh, Pennsylvania, USA

\begin{abstract}
An integrated capacitive humidity sensor on-chip with CMOS interface electronics is demonstrated to have a sensitivity of $0.31 \%$ change in capacitance per percent relative humidity, $72 \%$ higher than the highest previously demonstrated in an integrated sensor. The vertical parallel-plate sensor made in CMOS is a high sensitivity topology that had not been previously integrated. The sensitized dielectric between two metal electrodes is formed by first forming a cavity by etching an intermediate layer of metal in the CMOS stack and then filling the cavity with polymer through inkjet deposition. This topology is demonstrated with polyimide to make a humidity sensor, but with appropriate polymer selection can also be used to sense other chemicals.
\end{abstract}

\section{INTRODUCTION \\ Background}

A capacitive chemical sensor consists of a pair of electrodes across a dielectric layer that changes its dimensions or dielectric constant when it absorbs analyte, resulting in a change in capacitance. Water has a dielectric constant of approximately 78 at room temperature while most polymers have dielectric constants below 10, resulting in a large change in capacitance when water vapor is absorbed. Capacitive sensing is also a good candidate for an integrated humidity sensor because the metal layers available in CMOS can be used as electrodes to minimize fabrication cost.

One possible technique for integrating a capacitive sensor would be to deposit polymer on the exposed top metal layer of a CMOS chip, forming the structure shown in Fig. 1(a). However, oxide is in the most direct path for electric field between the electrodes, resulting in a large oxide capacitance in parallel to the capacitance through the polymer. Since only the polymer capacitance changes upon humidity exposure, the fractional sensitivity of the capacitor will be very small. In a non-planarized CMOS process, the electrodes can be structured by having one electrode consist of a two-metal stack (Fig. 1(b)) to obtain a larger polymer capacitance ([1], [2]). A large oxide capacitance remains, however; in [2] the polymer capacitance is only $18 \%$ of the total capacitance, resulting in a large degradation in sensitivity. The oxide directly between the electrodes and the substrate underneath can also be etched away, allowing polymer to fill the space directly between the electrodes (Fig. 1(c)) [3]. This design gives the highest sensitivity previously demonstrated in an integrated capacitive humidity sensor $(0.18 \%$ change in capacitance per percent relative humidity), but a significant parallel capacitance through the air above and below the electrodes remains, reducing the sensitivity.

In non-integrated capacitive sensors, a vertical parallel-plate structure (Fig. 1(d)) [4] is commonly used. The sensor consists of a layer of polymer sandwiched between two electrode layers; the top electrode is patterned to allow water vapor to absorb into the polymer layer. Since all of the electric field lines must pass through the polymer layer, the vertical parallel-plate sensor gives the highest possible sensitivity for a given polymer layer. The sensitivity for polyimide was measured to be $0.31 \% / \% \mathrm{RH}$ [4]. This paper presents the successful monolithic integration of a high sensitivity vertical parallel-plate structure with CMOS interface electronics.

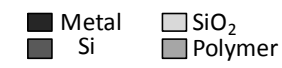

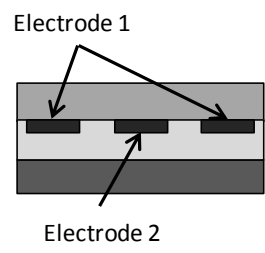

(a)

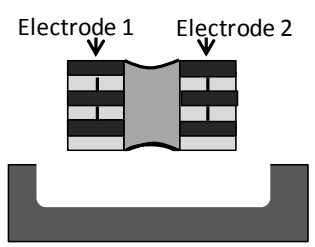

(c)

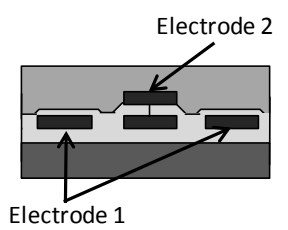

(b)

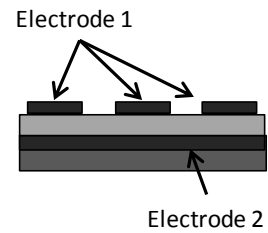

(d)
Figure 1: Capacitive chemical sensor designs

\section{Fabrication}

In order to obtain a vertical parallel-plate capacitor in a CMOS stack, a conductive layer must be obtained on either side of a cavity. One possibility, shown in Fig. 2(a), is to use two metal layers as electrodes, while etching the metal layer in between to form the cavity. This technique results in an oxide capacitance above and below the polymer layer due to the inter-metal dielectrics, reducing the sensitivity. However, in many CMOS processes, the metal layers consist of TiW adhesion layers above and below aluminum. By using a selective aluminum etch, these TiW layers can be left intact, and can be used as the top and bottom electrodes of a vertical parallel-plate sensor, as illustrated in Fig. 2(b). Vias connect the adhesion layers to other metal layers that wire the sensor to interface circuitry.

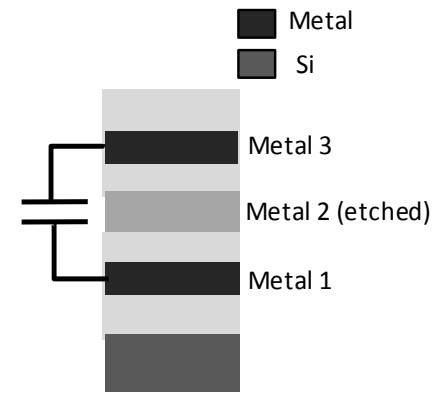

(a)

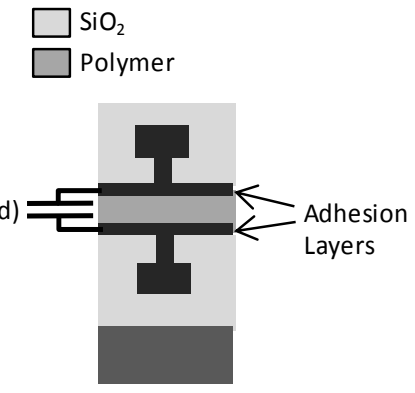

(b)
Figure 2: Options for integrating vertical parallel-plate sensor

The fabrication steps necessary to create the sensor are shown in Fig. 3. The process begins with a CMOS chip fabricated using the Jazz Semiconductor $0.35 \mu \mathrm{m}$ process (Fig. 3(a)). An anisotropic oxide etch is performed down to the second lowest metal layer (Fig. 2(b)). The aluminum part of this layer is then wet etched (Transense Aluminum Etchant Type A), leaving the TiW 
layers intact (Fig. 2(c)). Photoresist is patterned to protect the aluminum bondpads and the topmost metal layer is used as a mask to protect the first and third metal layers from the aluminum etch. The chip is rinsed in DI water, followed a rinse in acetone and a final rinse in methanol. This is necessary to avoid surface tension pulling the cavity closed. Polymer is then added in solution using inkjet deposition into an access hole in the center of the structure, with capillary forces pulling the polymer solution into the structure (Fig. 3(d)). The polymer used was a formulation of polymide in solution (HD Microsystems PI 2556) that was further diluted by a factor of 24:1 with a 50:50 solvent mixture of n-methyl-2pyrrolidone and methoxy propanol (HD Microsystems T-9039) to obtain a low enough viscosity to deposit with the inkjet. A similar fill structure in a non-integrated sensor was made previously using the MUMPS process [5].

\section{Metal $\square \mathrm{SiO}_{2}$ Si $\square$ Polymer}

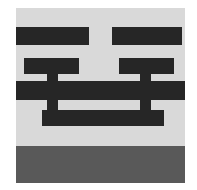

(a)

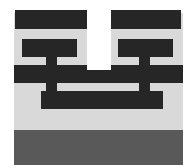

(b)

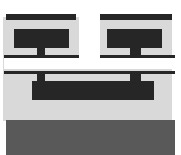

(c)

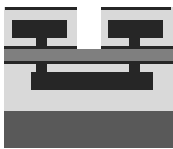

(d)
Figure 3: Fabrication process flow

\section{Design}

A cross section of the sensor design is shown in Fig. 4 a. A $300 \mu \mathrm{m}$ diameter circular capacitive sensor was fabricated using the above process. An $80 \mu \mathrm{m}$ diameter circular access hole in the center of the device is the inkjet target. Multiple square holes $(2.6 \mu \mathrm{m}$ on a side) act both as release holes and to allow water vapor to absorb more easily into the polymer layer. Oxide pillars are placed around the center hole to hold the plates apart (Fig. 4b).

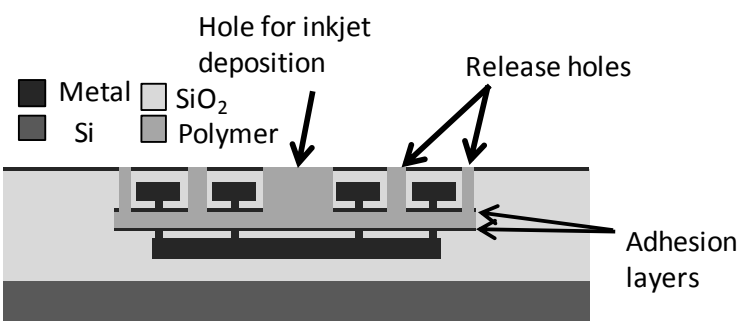

(a)

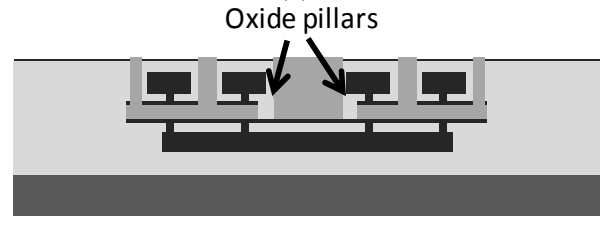

(b)

Figure 4: (a) Cross section of integrated sensor and (b) cross section that passes through oxide pillars
SEM images of the sensor before and after polymer jetting are shown in Fig. 5. Since it is difficult to judge optically whether the sensor is full of polymer, the structure was deliberately overloaded, resulting in a polymer residue on the top surface of the chip (Fig. 5(b)). This residue layer will reduce the speed of the device slightly, since water vapor will need to diffuse a longer distance into the device to reach the dielectric in the cavity. However, the process could be optimized to minimize the excess polymer and improve the response time.

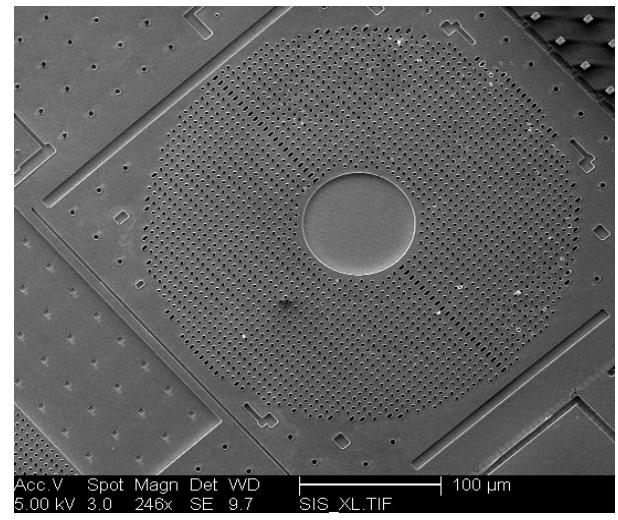

(a)

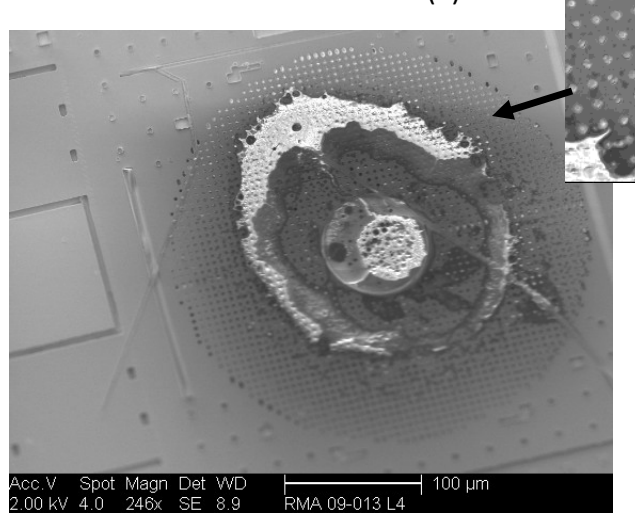

(b)

Figure 5: SEM image of sensor (a) before and (b) after inkjetting of polymer with inset picture of filled release holes

\section{Theoretical Model}

The humidity sensor is modeled using a parallel-plate capacitor approximation, since the gap between the electrodes (nominally $450 \mathrm{~nm}$ ) is very small relative to the area of the structure. The dielectric constant for polymer with absorbed water vapor is [6]

$$
\varepsilon=\left[\gamma\left(\varepsilon_{\mathrm{H}_{2} O} \mathrm{O}^{1 / 3}-\varepsilon_{P}^{1 / 3}\right)+\varepsilon^{1 / 3}\right]^{3}
$$

where $\gamma$ is the volume fraction of water in the film, and $\varepsilon_{P}$ and $\varepsilon_{H 2 O}$ are the dielectric constants of polymer and water, respectively. For humidity sensing, the most common polymer used is polyimide due to its linear response to relative humidity. Polyimide has a volume fraction of water approximately given by [6]

$$
\gamma=c_{1}(\% R H)^{c_{2}}
$$

The terms $c_{1}$ and $c_{2}$ are temperature-dependent constants. 


\section{TESTING}

\section{Testing Circuit}

The sensor was integrated with the charge-based capacitance measurement (CBCM) circuit shown in Fig. 6 [7]. This circuit uses two separate measurements to isolate the parasitic capacitance of the wiring and circuit from the sensor capacitance. In the first measurement, the Clk input is grounded while the center node is first discharged to ground and then brought up to $V_{d d}$. The charge during this measurement is

$$
Q_{1}=V_{d d}\left(C_{p}+C\right)
$$

In the second measurement, the Clk input is held at ground while the center node is discharged, then brought to $V_{d d}$ when the node is charged. This results in no voltage drop across the sensing capacitance during the charging phase, giving a charge equal to:

$$
Q_{2}=V_{d d} C_{p}
$$

The charge necessary to charge the center node in each measurement is measured by repeating the charging at a set frequency $f$ and measuring the current with a DC picoammeter. The sensor capacitance is found by the expression:

$$
C=\frac{I_{1}-I_{2}}{f V_{d d}}
$$

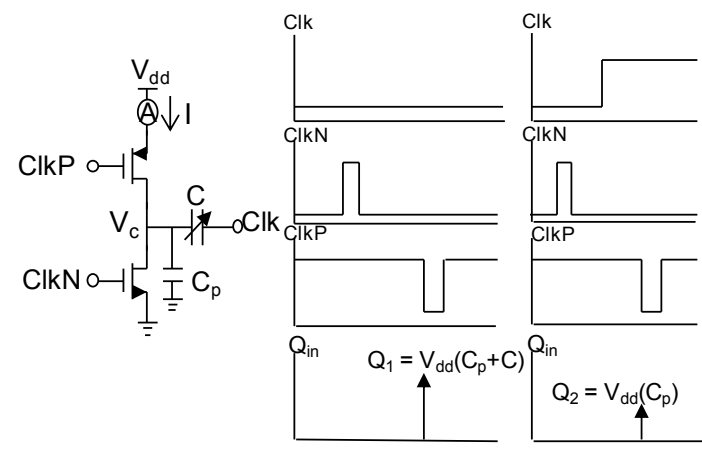

(a)

(b)

(c)

Figure 6: (a) CBCM circuit and timing diagrams for (b) first measurement and (c) second measurement

\section{Flow System}

The sensor was tested using the flow system shown in Fig. 7. A Milligat M6 solvent pump pumps liquid water into a $1 \mathrm{~L} / \mathrm{min}$ nitrogen flow; a brass block heated to $48^{\circ} \mathrm{C}$ is used to encourage the water to vaporize. The airflow passes into a $250 \mathrm{~mL}$ mixing volume, and then into the test box containing the sensor. A Honeywell HIH 4000 humidity sensor in the test box is used to obtain actual reference humidity value.

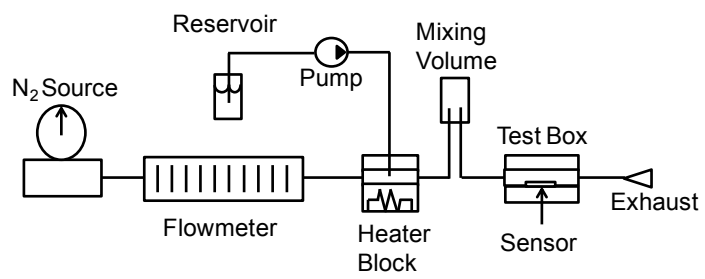

Figure 7: Humidity sensor test setup

\section{RESULTS \\ Humidity Testing}

The sensor was measured for a range of relative humidity from $0 \%$ to $40 \%$, resulting in the response curve in Fig. 8 . The sensor response was fitted to the theoretical model for polyimide, giving an adjusted $\mathrm{R}^{2}$ value of 0.9964 . The sensitivity in the linear region of the sensor was fitted to be $0.31 \%$ change in capacitance per percent relative humidity.

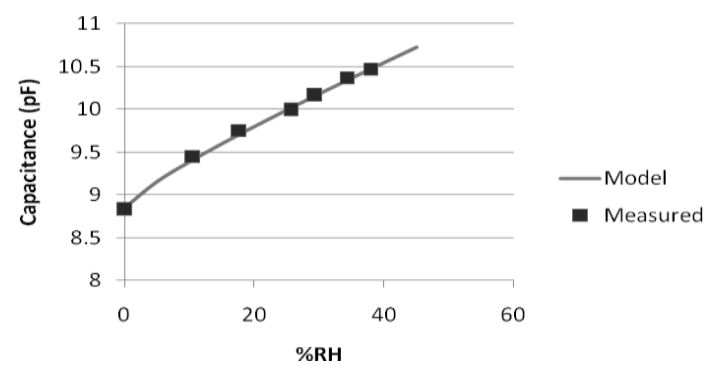

Figure 8: Humidity sensor response curve

\section{Response Time}

In the integrated vertical parallel-plate structure, polymer wicks to the top of the release holes, as shown schematically in Fig. 4. As a result, water vapor must diffuse through several microns of polymer before reaching the area between the two plates that forms the actual capacitor, potentially resulting in a slow response time. Fig. 9 shows result from a finite-element simulation of the response time to a humidity step of $3 \%$ change in relative humidity using the diffusion model in [8] and a diffusion coefficient for water vapor in polyimide of $15 \times 10^{-14} \mathrm{~m}^{2} / \mathrm{s}$ [9]. The simulated time constant of the sensor is $68 \mathrm{~s}$.

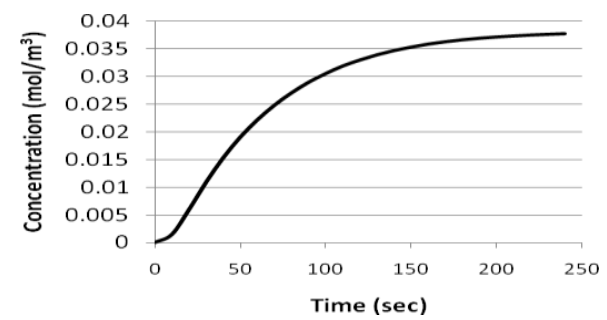

Figure 9: Simulation of humidity concentration between plates in response to stepped humidity pulse

In order to verify the response time, the sensor was compared to the reference Honeywell humidity sensor, which has a response time constant to changes in relative humidity of $15 \mathrm{~s}$ [10]. Fig. 10 shows the response to both sensors to two pulses of humidity with different amplitudes. As expected, the sensor was noticeably slower than the reference sensor; this effect was particularly pronounced in the falling portion of the humidity pulse.

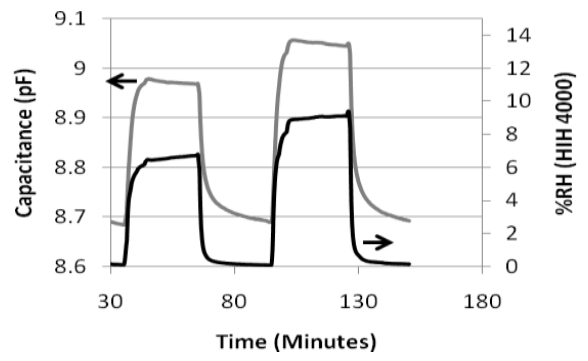

Figure 10: Sensor response to humidity pulses 


\section{Noise}

Another important specification of a chemical sensor is the noise performance. One method for characterizing the noise response and limit of detection of a sensor is the Allan variance:

$$
\sigma_{C}^{2}=\frac{1}{M} \sum_{k=1}^{M} \frac{1}{2}\left(C_{k}-C_{k-1}\right)^{2}
$$

where $M$ is the number of samples. $C_{i}$ is the $i^{\text {th }}$ measured capacitance value, normalized by the initial capacitance $C_{0}$. Since the Allan variance measures the sample to sample variation, it can be used to calculate an approximate minimum detectable capacitance change,

$$
\Delta C=C_{0} \sqrt{\sigma_{C}^{2}}
$$

The Allan variance is dependent on the sampling period, since averaging samples together reduces the variation from higher frequency noise. At longer averaging times, however, low frequency drift causes the Allan variance to rise. A plot of the Allan variance versus averaging time of the sensor is shown in Fig. 11.

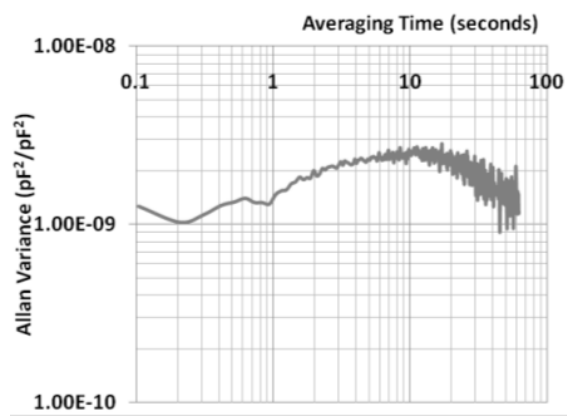

Figure 11: Allan variance of sensor

As shown, the Allan variance is flat over the averaging times tested. The lowest Allan variance was $1.023 \times 10^{-9} \mathrm{pF}^{2} / \mathrm{pF}^{2}$ for an averaging time of $0.2 \mathrm{~s}$. The minimum detectable signal is $0.26 \mathrm{fF}$, corresponding to a limit of detection of $0.01 \%$ relative humidity.

\section{Temperature Response}

The response of the sensor to changes in temperature was also measured (Figure 12), giving a sensitivity of $0.19 \%$ change in capacitance per ${ }^{\circ} \mathrm{C}$. The measured sensitivity to temperature is 2.7 times higher than the sensitivity of the released interdigitated sensor measured in [3]. The high sensitivity to temperature may be because the low density of the oxide pillars in the current structure allows the top capacitor plate to move slightly as temperature changes. A design using a larger number of pillars to hold the plate distance constant is currently being fabricated.

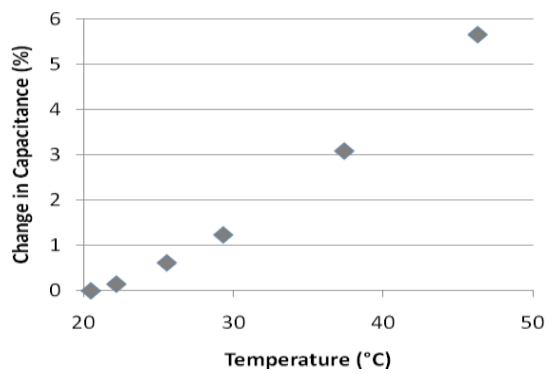

Figure 12: Temperature response

\section{CONCLUSION}

The measured results demonstrate a vertical parallel-plate capacitive humidity sensor successfully integrated with CMOS testing electronics. The sensitivity of the sensor is $0.31 \% / \% \mathrm{RH}$, an increase of $72 \%$ over the highest sensitivity previously demonstrated for an integrated capacitive humidity sensor [3]. The sensitivity matches the sensitivity for polyimide previously measured in a non-integrated vertical parallel-plate sensor [4]. The sensor was found to be relatively slow, with a time constant on the order of a minute, since water vapor must diffuse several microns to reach the active part of the sensor. The limit of detection of the sensor was found to be $0.01 \%$ relative humidity.

\section{ACKNOWLEDGEMENT}

The authors thank Lawrence Schultz and Lee Weiss for advice and assistance with inkjetting and Suresh Santhanam for performing the oxide etch. This work is funded in part by NIOSH/CDC (contract 200-2002-00528), in part by AFOSR FA9550-07-1-0245, and in part by the U.S. Department of Homeland Security under an Interagency Agreement with the Centers for Disease Control. The findings and conclusions in this publication have not been formally disseminated by the National Institute of Occupational Safety and Health, the U.S. Department of Homeland Security, or the U.S. Office of Scientific Research and should not be construed to represent any agency determination or policy.

\section{REFERENCES}

[1] C. Hagleitner, D. Lange, A. Hierlemann, O. Brand, and H. Baltes, "CMOS single-chip gas detection system comprising capacitive, calorimetric and mass-sensitive microsensors," IEEE J. Solid-State Circuits, vol. 37, no. 12, pp. 1867-1878, Dec. 2002.

[2] A. Hierlemann, Integrated Chemical Microsensor Systems in CMOS Technology, Berlin, Germany: Springer-Verlag, 2005.

[3] N. Lazarus, S. S. Bedair, C.-C. Lo, G. K. Fedder, "CMOSMEMS capacitive humidity sensor," J. Microelectromech. Syst., vol.19, no. 1, pp. 183-191, Feb. 2010.

[4] M. Dokmeci and K. Najafi, "A high sensitivity polyimide capacitive relative humidity sensor for monitoring anodically bonded hermetic micropackages," J. Microelectromech. Syst., vol. 10, no. 2, pp. 197-204, June 2001.

[5] S. V. Patel, T. E. Mlsna, B. Fruhberger, E. Klaassen, S. Cemalovic and D. R. Baselt, "Chemicapacitive microsensors for volatile organic compound detection," Sensors and Actuators B, vol. 96 no. 1 pp. 541-553, Dec. 2003.

[6] H. Shibata, M. Ito, M. Asakura, and K. Watanabe, "A digital hygrometer using a capacitance to frequency converter," in Proc. of IEEE IMTC 1995, Waltham, MA, April 1995, pp. 100-106.

[7] Y. Chang, H. Chang, T. Lu, Y. King, W. Ting, Y. Ku and C. $\mathrm{Lu}$, "Charge-based capacitance measurement for biasdependent capacitance," IEEE Electron Device Letters, Vol. 27, No. 5, pp. 390-392, 2006.

[8] A. Tetelin and C. Pellet, "Accurate model of the dynamic response of a capacitive humidity sensor," in Proc. IEEE Sensors, Toronto, ON, Canada, 2003, pp. 378-383.

[9] J. Seo and H. Han, "Water Diffusion studies in polyimide thin films," J. Appl. Polym. Sci., vol. 82, no. 3, pp. 731-737, Oct. 2001.

[10] Honeywell, "HIH-4000-01”, HIH-4000 datasheet, July 2006.

\section{CONTACT}

*N. Lazarus, tel: +1-412-268-4403; nlazarus@cmu.edu 